\title{
Studies of an Intergalactic HI Cloud
}

\author{
Jayanne English \\ University of Manitoba, Canada \\ B. Koribalski \\ Australia Telescope National Facility, CSIRO, Australia
}

\author{
K.C. Freeman \\ Research School of Astronomy and Astrophysics, The Australian \\ National University, Australia
}

\begin{abstract}
.
An intergalactic $\mathrm{HI}$ cloud of a few $\times 10^{9} \mathcal{M}_{\odot}$, previously detected using the Parkes Radio Telescope and the Australia Telescope Compact Array (ATCA) (English 1994; Freeman et al. 1996), has been confirmed in further ATCA observations of the NGC 3256 galaxy group. The group contains the prominent merging galaxy NGC 3256, which is surrounded by a number of HI fragments (English et al. 2003), the tidally disturbed galaxy NGC 3263 (Koribalski et al., in prep.), and several other galaxies. Using ATCA HI data we examine the nature of this massive gas cloud and its relationship to the neighbouring galaxies. This could be a primordial "galaxy building block". However the cloud's properties, in conjunction with the spatial extents and velocity behaviours of the group's major galaxies, may indicate that it originated out of tidal debris.
\end{abstract}

The most spectacular feature in the NGC 3256 Group is a galaxy-sized HI cloud (see Figure 1 and the front cover illustration of the book). Covering an area of about $100 \mathrm{kpc}(\mathrm{EW}) \times 175 \mathrm{kpc}(\mathrm{NS})$, our analysis of an ATCA HI velocity cube $\left(84^{\prime \prime} \times 67^{\prime \prime}\right.$ synthesized beam; $6.5 \mathrm{~km} \mathrm{~s}^{-1}$ velocity resolution) shows that the cloud contains at least $3 \times 10^{9} \mathcal{M}_{\odot}$. A Parkes Telescope spectrum indicates it could have an HI mass of $5 \times 10^{9} \mathcal{M}_{\odot}$.

This cloud is less likely to be primordial and more likely to have had its origins in tidal disturbances, since it is not obviously isolated from its neighbouring galaxies. Situated just west of NGC 3263, it has heliocentric velocities that are similar to those of NGC 3256. Regardless of its origin, a question to consider is the fate of this gas. Our initial characterization considers whether the cloud could become a source of dwarf galaxies and/or extra-galactic HII regions. The following are our preliminary results.

- The cloud's substructure includes 6 flux density enhancements worth examining for their potential to form dwarf galaxies and other bound stellar objects.

- One of the HI enhancements has possibly produced a dwarf galaxy. The position of starbursting galaxy, WPV060 (Wamsteker et al. 1985) is within the beam 


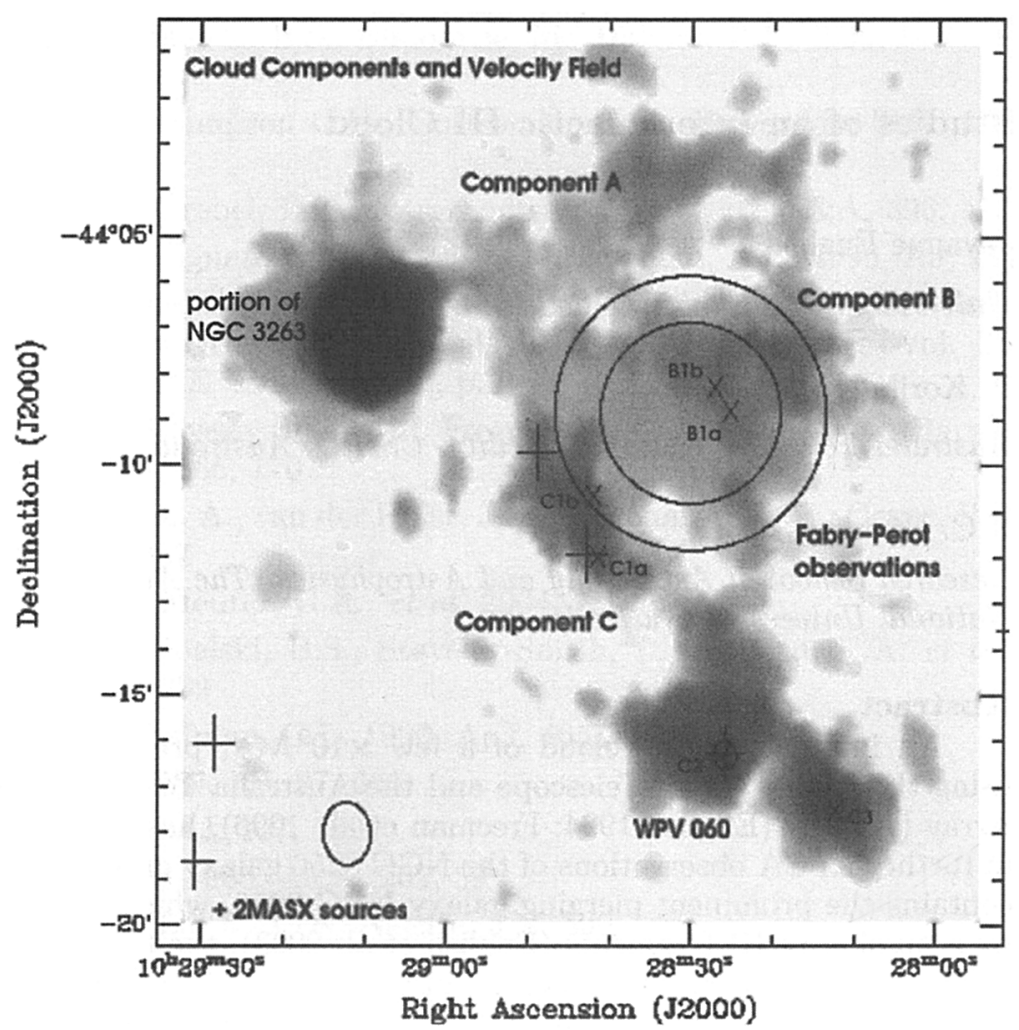

Figure 1. Features in the Velocity Field. This image combines three velocity ranges: 2744.9 through $2794.1 \mathrm{~km} \mathrm{~s}^{-1}, 2803.8-2823.5 \mathrm{~km} \mathrm{~s}^{-1}$; and $2833.39-2902.2 \mathrm{~km} \mathrm{~s}^{-1}$. The most blueshifted velocities are in Component C (stretching from NGC 3263 towards the SW); the most redshifted velocities are in B. X marks positions of enhancements in HI spectra; crosses are 2MASX sources. WPV060 (ellipse) is a starburst galaxy. At a distance of $37.6 \mathrm{Mpc}, 1$ arcmin equals $11 \mathrm{kpc}$.

of the position of the enhancement's peak flux. This galaxy's velocity of 2760 $\mathrm{km} \mathrm{s}^{-1}$ (Winkler et al. 1992) is also consistent with the HI enhancement's heliocentric velocity range; the peak flux occurs at $2786 \mathrm{~km} \mathrm{~s}^{-1}$ and the enhancement has a Full-width Half-maximum of $49 \mathrm{~km} \mathrm{~s}^{-1}$.

$-\mathrm{H}_{\alpha}$ emission was not detected in a region delineated by the annulus ( $1^{\prime}$ wide; $1^{\prime}$ radius) of the TAURUS Fabry-Perot on the Anglo-Australian Telescope in a study by Bland-Hawthorne, McCain, and Freeman. Thus this portion of the cloud is unlikely to be ionized by the UV radiation field of NGC 3263 or by strong shocks that would be created by colliding gas. The integration time of 2 hours was not sufficient to detect ionization by the cosmic UV background. Since this region was selected using preliminary $\mathrm{HI}$ data, more $\mathrm{H}_{\alpha}$ observations are required. 
We are also studying neighbouring galaxies NGC 3256 and NGC 3263. NGC 3256 appears to have $10^{\prime \prime}$ diameter flux density enhancements in its tidal tails and in its detached gas fragments. NGC 3263 displays a $30 \mathrm{kpc}$ long tidal tail which has a perpendicular extension devoid of stars.

\section{References}

English, J., Norris, R. P., Freeman, K. C., \& Booth, R. S. 2003, AJ, 125, 1124 English 1994, Ph.D. Thesis

Freeman, K. C., English, J., \& Norris, R. P. 1996, JRASC, 90, 333

Wamsteker, W. et al., 1985, Astr. Ap. Suppl., 62, 255

Winkler, H., Stirpe, G. M., \& Sekiguchi, K. 1992, A\&AS, 94, 103 